PROCEEIDIN(;S OF THE

AMERICAN MATHEMATICAL SOCIETY

Volume 87 . Number 1. January 1983

\title{
A CONVERSE TO THE LUSIN-PRIVALOV RADIAL UNIQUENESS THEOREM
}

\author{
ROBERT D. BERMAN
}

\begin{abstract}
Let $E$ be a subset of the unit circumference $C$. If for every nonempty open $\operatorname{arc} A$ of $C$, the set $E$ is not both metrically dense and of second category in $A$, then there exists a nonconstant analytic function $f$ on the open unit disk $\Delta$. such that $f^{*}(\eta)=0, \eta \in E$. where $f^{*}$ is the radial limit function of $f$.
\end{abstract}

Let $\Delta=\{|z|<1\}$ and let $E$ be a subset of $C=\{|=|=1\}$. For $f$ an analytic function on $\Delta$, denote by $f^{*}$ the radial limit function of $f$. Thus $f^{*}(\eta)=\lim _{r-1} f(r \eta)$ for each $\eta$ in $C$ where the limit exists (finite or infinite). According to a classical theorem of Fatou [2], a bounded analytic function $f$ on $\Delta$ has radial limits almost everywhere in $C$. F. and M. Riesz [6] proved that if there exists a nonconstant bounded analytic function $f$ on $\Delta$ such that $f^{*}(\eta)=0, \eta \in E$, then $E$ is of measure 0 . A converse to the Riesz theorem was provided by Privalov [5, p. 214].

THEOREM 1. If $E$ is of measure 0 , then there exists a nonconstant bounded analytic function $f$ on $\Delta$ such that $f^{*}(\eta)=0, \eta \in E$.

Here we are concerned with a converse to the following celebrated uniqueness theorem of Lusin and Privalov [3, pp. 187-189].

THEOREM 2. If there exists a nonconstant analytic function $f$ on $\Delta$ such that $f^{*}(\eta)=0, \eta \in E$, then for every nonempty open arc $A$ in $C$, the set $E$ is not both metrically dense and of second category in $A$.

By definition $E$ is metrically dense in an open arc $A$ if, for every nonempty open subarc $B$ of $A$, the set $E \cap B$ has positive outer measure. Also, $E$ is nowhere dense if int $\bar{E}=\varnothing$, that is, the interior of the closure of $E$ is empty, and is of first (resp. second) category if it is (resp. is not) a countable union of nowhere dense sets.

Lusin and Privalov [3, $\$ 11$ and 33-35] constructed nonconstant analytic functions $f$ and $g$ such that $f^{*}(\eta)=0$ for a set of $\eta$ in $C$ of measure $2 \pi$ and $g^{*}(\eta)=0$ for a set of $\eta$ in $C$ which is of measure $\pi$ in the upper half-circle and of second category in the lower half-circle. These examples show that some nontrivial combination of the second category and metric density conditions in Theorem 2 is indeed necessary.

In this paper, the full converse of Theorem 2 is proved.

Received by the editors May 3, 1982.

1980 Mathematics Subject Classification. Primary 30D40.

Key words and phrases. Lusin-Privalov, radial uniqueness. 
THEOREM 3. If, for every nonempty open arc $A$ in $C$, the set $E$ is not both metrically dense and of second category in $A$, then there exists a nonconstant analytic function $f$ on $\Delta$ such that $f^{*}(\eta)=0, \eta \in E$.

We shall need the following decomposition lemma.

LEMMA 1. Suppose that for every nonempty open arc $A$ in $C$, the set $E$ is not both metrically dense and of second category in $A$. Then there exists a closed subset $F$ of $C$ such that $E \cap F$ is of first category and $E \backslash F$ is of measure 0.

Proof. Let $F$ be the set of $\eta$ in $C$ such that for every open $\operatorname{arc} A$ containing $\eta$, the set $E \cap A$ has positive outer measure. Evidently $F$ is closed.

$E \cap F$ is of first category. Since $F=(F \backslash$ int $F) \cup$ int $F$ and $F \backslash$ int $F$ is nowhere dense, it suffices to check that $E \cap$ int $F$ is of first category. Putting aside the trivial case, assume that int $F \neq \varnothing$. Let $A$ be a component (open arc) of int $F$. We claim that $E$ is metrically dense in $A$. If not, there exists some nonempty open subarc $B$ of $A$ such that $E \cap B$ has measure 0 . But this contradicts the fact that $B \subset F$ and the definition of $F$, so the claim is verified. Since, by assumption, $E$ is not both metrically dense and of second category in $A$, we conclude that $E \cap A$ is of first category. The required conclusion now follows from the fact that int $F$ has at most countably many components.

$E \backslash F$ is of measure 0 . If $F=C$ the assertion is trivial, so assume $C \backslash F \neq \varnothing$. By the definition of $F$, there exists for each $\eta$ in $C \backslash F$ an open $\operatorname{arc} A_{\eta}$ containing $\eta$ such that $E \cap A_{\eta}$ has zero measure. By the countable basis property of $C$, the open cover $\left(A_{\eta}\right)_{\eta \in C \backslash F}$ of $C \backslash F$ has a countable subcover $\left(A_{\eta_{k}}\right)_{1}^{\infty}$, where $\left\{\eta_{k}\right\}_{1}^{\infty} \subseteq C \backslash F$. Now $E \cap A_{\eta_{k}}$ has zero measure for each $k$, so the same follows for $E \backslash F$ since $E \backslash F \subseteq$ $\cup_{1}^{\infty}\left(E \cap A_{\eta_{k}}\right)$.

Lemma 1 is established.

We shall also require a slightly strengthened form of a theorem of Bagemihl and Seidel [1].

THEOREM 4. If $E$ is of first category, then there exists a nonconstant analytic function $f$ on $\Delta$ which is analytic at each point of $C \backslash \bar{E}$ and satisfies $f^{*}(\eta)=0, \eta \in E$.

The proof of Theorem 4 that we shall give, except for the modification used here to guarantee that $f$ is analytic in $C 、 \bar{E}$, follows the outline given by Schneider in [7, p. 335].

Proof. If $E=\varnothing$, let $f(z)=\exp (z), z \in \Delta$. Assume now that $E \neq \varnothing$. Let $R$ be the region $\Delta \cup\{0<|z|<2, z /|z| \in C \backslash \bar{E}\}$ and $\left(F_{n}\right)_{1}^{\infty}$ a monotone nondescending sequence of closed nowhere dense sets such that $E \subseteq \cup_{1}^{\infty} F_{n} \subseteq \bar{E}$. Define $W_{n}=\{\eta$ $\in C: \operatorname{dist}(\eta, \bar{E}) \geqslant 1 / n\}$ for each positive integer $n$, where

$$
\operatorname{dist}(z, W)=\inf _{w \in W}|z-w| \text { for } z \in \mathbf{C}, W \subseteq \mathbf{C},
$$


and note that $\left(W_{n}\right)_{1}^{\infty}$ is a monotone nondescending sequence of compact subsets of $C \backslash \bar{E}$ such that $\cup_{1}^{\infty}$ int $W_{n}=C 、 \bar{E}$. Let $S_{n}=\{|z| \leqslant 1-1 / n\} \cup\{0<|z| \leqslant 2$, $\left.z /|z| \in W_{n}\right\}$ and $T_{n}=\left\{1-1 / n \leqslant|z| \leqslant 1, z /|z| \in F_{n}\right.$ when $\left.z \neq 0\right\}$, and define

$$
h_{n}(z)= \begin{cases}0, & z \in S_{n}, \\ n 2^{n}[|z|-(1-1 / n)], & z \in T_{n},\end{cases}
$$

for all positive integers $n$.

Since $h_{n}$ is continuous on the compact set $K_{n}=S_{n} \cup T_{n}$ and analytic in its interior, and $C \backslash K_{n}$ is connected, Mergelyan's Theorem [4] implies the existence of a polynomial $p_{n}$ such that

$$
\left|p_{n}(z)-h_{n}(z)\right|<1 / 2^{n}, \quad z \in K_{n},
$$

for every positive integer $n$. Noting that for each compact subset $K$ of the region $R$ there exists an $n$ such that $K \subseteq S_{n}$, and that this implies $h_{n}(z)=0, z \in K$, by (1), we see from (2) and the Weierstrass $M$-test that $\Sigma_{1}^{\infty} p_{n}$ converges uniformly on compact subsets of $R$ to a function $g$ analytic on $R$.

Let $f$ be the restriction of $\exp (-g)$ to $\Delta$. Then $f$ is a nonvanishing analytic function on $\Delta$ which is analytic at each point of $C 、 \bar{E}$. We claim that $f^{*}(\eta)=0, \eta \in E$. It suffices to show that $\lim _{r \rightarrow 1} \operatorname{Re} g(r \eta)=+\infty, \eta \in E$. Let $\eta \in E$. Then there exists a positive integer $n$ such that $\eta \in F_{j}$ for $j \geqslant n$. If $m \geqslant n$, it follows from (1) and (2) that

$$
\begin{aligned}
\operatorname{Re} g(r \eta) & \geqslant 2^{m-1}-\sum_{\substack{j \geqslant n \\
j \neq m}} 2^{-j}-\sum_{1}^{n-1}|p(r \eta)| \\
& \geqslant 2^{m-1}-\alpha, \quad 1-1 /(2 m) \leqslant r<1,
\end{aligned}
$$

where $\alpha$ is a positive constant not depending on $m$. The required conclusion follows and the claim is verified. Finally, $f$ is nonconstant since it is nonvanishing and $f^{*}(\eta)=0, \eta \in E \neq \varnothing$. The proof is completed.

We are now ready to prove Theorem 3 .

Proof of Theorem 3. Assume $E \neq \varnothing$. Take $F$ as in Lemma 1. Since $E \backslash F$ has zero measure, there exists, by Theorem 1 , a nonconstant bounded analytic function $g$ on $\Delta$ such that $g^{*}(\eta)=0, \eta \in E \backslash F$. Since $E \cap F$ is of first category, Theorem 4 implies there exists a nonconstant analytic function $h$ on $\Delta$ such that $h$ is analytic at each point of $C \backslash(\overline{E \cap F}) \supseteq C \backslash F$ and $h^{*}(\eta)=0, \eta \in E \cap F$.

Let $f=g h$. Then $f$ is analytic on $\Delta$. From the condition $g^{*}(\eta)=0, \eta \in E \backslash F$, and the analyticity of $h$ at each point of $C \backslash F$, we have $f^{*}(\eta)=0, \eta \in E \backslash F$. On the other hand, since $h^{*}(\eta)=0, \eta \in E \cap F$, and $g$ is bounded, we conclude that $f^{*}(\eta)=0, \eta \in E \cap F$. Thus $f^{*}(\eta)=0, \eta \in(E \backslash F) \cup(E \cap F)=E$. The nonconstancy of $f$ follows from the fact that it is a product of nonconstant functions and that $f^{*}(\eta)=0, \eta \in E \neq \varnothing$. This completes the proof.

We note that since the functions constructed by Privalov for Theorem 1 and here for Theorem 4 are nonvanishing, the function $f$ of Theorem 3 may also be required to be nonvanishing. 


\section{REFERENCES}

1. F. Bagemihl and W. Seidel, Some boundary properties of analytic functions, Math. Z. 61 (1954), 186-199.

2. P. Fatou, Séries trigonométriques et séries de Taylor, Acta Math. 30 (1906), 335-400.

3. N. N. Lusin and I. I. Privalov, Sur l'unicité et la multiplicite des fonctions analytiques, Ann. Sci. École Norm. Sup. (3) 42 (1925), 143-191.

4. S. N. Mergelyan. Amer. Math. Soc. Transl. 3 (1962), 287-293.

5. I. I. Privalov, Randeigenschaften analytischer Funktionen, VEB Deutscher Verlag der Wissenschaften, Berlin, 1956.

6. F. Riesz and M. Riesz, Über die Randwerte einer analytischen Funktion, Quatrième Congrès des Math. Scand. Stockholm, 1916, pp. 27-44.

7. W. J. Schneider, Approximation and harmonic measure, Aspects of Contemporary Complex Analysis (D. H. Brannan and J. G. Clunie, eds.), Academic Press, New York, 1980, pp. 334-340.

Department of Mathematics, University of Maryland, College Park, Maryland 20742

Current address: Department of Mathematics, Wayne State University, Detroit, Michigan 48202 